\title{
Clinical outcomes following high-dose-rate surface applicator brachytherapy for angiosarcoma of scalp and face
}

\author{
Devarati Mitra, MD, PhD',2, Phillip M. Devlin, MD', Ivan Buzurovic, PhD', Katherine Thornton, MD³, Allen C. Lam, MD4, \\ Chandrajit P. Raut, MD 5 ,6, Elizabeth H. Baldini, MD 1,5 , Miranda B. Lam, MD, MBA',5 \\ 'Department of Radiation Oncology, Brigham and Women's Hospital and Dana-Farber Cancer Institute, Boston, MA, USA, ${ }^{2}$ Division of Radiation \\ Oncology, MD Anderson Cancer Center, Houston, TX, USA, ${ }^{3}$ Department of Medical Oncology, Memorial Sloan Kettering Cancer Center, \\ New York City, NY, USA, “Department of Otolaryngology. Massachusetts Eye and Ear Infirmary, Boston, MA, USA, ${ }^{5}$ Sarcoma and Bone Cancer \\ Treatment Center, Dana-Farber Cancer Institute, Boston, MA, USA, 'Department of Surgery, Brigham and Women's Hospital and Dana-Farber \\ Cancer Institute, Boston, MA, USA
}

\begin{abstract}
Purpose: Angiosarcoma is a sub-type of soft tissue sarcoma, often presenting as a multifocal or diffuse disease process with poor prognosis. This study presents outcomes of a single institution cohort of patients with angiosarcoma of the scalp and face following treatment with multimodality therapy, including high-dose-rate surface applicator (HDR-SA) brachytherapy, and represents the largest cohort utilizing this therapeutic approach.

Material and methods: Twenty patients with primary or recurrent angiosarcoma of the face or scalp were treated with HDR-SA brachytherapy between 2003-2018, with clinical characteristics and outcomes collected from medical records and used to identify prognostic features.

Results: Median follow-up was 45 months. Patients treated with HDR-SA brachytherapy had a 4-year local control rate of $63 \%$, a 4 -year progression-free survival (PFS) rate of $20 \%$, and a 4 -year overall survival rate of $54 \%$. Disease features associated with worse loco-regional control (LRC) included location on the scalp (vs. face, $p=0.04$ ) and tumor size $\geq 5 \mathrm{~cm}(p=0.0099)$. Outcomes after HDR-SA brachytherapy for salvage therapy vs. HDR-SA brachytherapy as a component of an initial treatment approach were also significantly different, with worse LRC $(p=0.0084)$ and worse overall survival (OS) $(p=0.0019)$ in a setting of salvage therapy.

Conclusions: Local control rates following HDR-SA brachytherapy for scalp or face angiosarcoma are moderate and similar to what is described in the literature using a variety of local control treatment modalities. Smaller tumors and those involving the face rather than scalp had better outcomes. PFS rates were poor and there is a pressing need for treatment intensification and novel therapeutic options.
\end{abstract}

J Contemp Brachytherapy 2021; 13, 2: 172-178 DOI: https://doi.org/10.5114/jcb.2021.105285

Key words: HDR brachytherapy, angiosarcoma.

\section{Purpose}

Angiosarcoma is a sub-type of soft tissue sarcoma originating from malignant transformation of endothelial cells [1]. Such tumors can arise anywhere in the body but most commonly present as cutaneous disease of the scalp and face [2]. Wide local excision alone is associated with a high-rate of local recurrence and high morbidity related to both treatment and disease recurrence [3-7]. Given the frequently diffuse and multifocal nature of cutaneous angiosarcoma, it is not uncommon for the disease to be unresectable at diagnosis. Systemic therapy is an attractive initial treatment modality and is employed for most angiosarcomas of the scalp and face at our institution. The optimal local treatment for this disease has not been determined and could include surgery, radiation therapy, or both modalities. Given the rarity of scalp and face angiosarcoma, prospective studies showing the benefit of radiation therapy are lacking, but several retrospective studies have demonstrated an improved local recurrence and survival with radiation treatment [6, 8-10].

There are multiple approaches to radiation therapy for face and scalp angiosarcoma, including older mixed lateral photon and electron approaches, modern photon radiotherapy techniques, such as intensity-modulated radiotherapy (IMRT) or volumetric-modulated arc ther-
Address for correspondence: Miranda B. Lam, MD, MBA, Department of Radiation Oncology, Brigham and Women's Hospital, 75 Francis St., Boston MA 02115, USA, phone: +1-857-307-3181, e-mail: Miranda_Lam@dfci.harvard.edu
Received: 10.11 .2020

Accepted: 07.01.2021

Published: 14.04 .2021 
apy (VMAT) as well as high-dose-rate surface applicator (HDR-SA) brachytherapy [11-17]. In our institutional practice, angiosarcoma of the face and scalp is treated with HDR-SA brachytherapy, but a comprehensive evaluation of clinical outcomes with this treatment approach was not performed. Theoretical advantages of this approach include the ability to conform a dose to often irregular, cutaneous target as well as the frequent ability to escalate a dose by delivering "hot spots" adjacent to the surface applicator, within tumor tissue.

We have recently published a study including dosimetric details of a subset of the patients from the current study who were treated with HDR-SA brachytherapy and had detailed planning information available [18]. The current study was a retrospective evaluation of the clinical characteristics and outcomes for the largest cohort, to our knowledge, of scalp and face angiosarcoma patients treated with HDR-SA brachytherapy, between 2003-2018.

\section{Material and methods}

\section{Patients' selection}

We identified 20 consecutive patients with histologically confirmed angiosarcoma of the scalp or face that were treated at our institution between 2003-2018 with HDR-SA brachytherapy. With institutional review board approval, these patients were extracted from institutional databases for subsequent review and analysis. All patient's medical records were reviewed after obtaining approval from our institutional review board.

\section{Therapeutic approach}

Our institutional practice in the management of scalp and face angiosarcoma has evolved over the 15 years included in this study, such that there was variability in the use of systemic therapy and surgery, but all patients in the cohort received HDR-SA brachytherapy. For HDRSA planning, computed tomography (CT) simulation was performed to treat the visualized tumor, with an addition of $5 \mathrm{~cm}$ radial clinical margin whenever anatomically feasible. Further technical details of HDR-SA brachytherapy planning have been described [18]. All, except one patient (treated in 2003), were prescribed 51 Gy in 17 fractions. This first patient was prescribed 51.2 Gy in 16 fractions.

\section{Clinical outcomes}

Electronic medical records of all patients were reviewed to assess clinical outcomes and treatment-associated toxicity. The two primary acute toxicities studied were dermatitis and alopecia, which were graded as per CTCAE v4.0. Local control (LC) was defined as absence of recurrence within the radiation field or at the margin ( $\leq 2 \mathrm{~cm}$ of the field edge). Local-regional control (LRC) was defined as absence of local recurrence or discontinuous cutaneous regional recurrence $(>2 \mathrm{~cm}$ from the field edge) or lymph node recurrence in the head and neck. Distant metastasis was defined as recurrence outside the head and neck area.

\section{Statistical analyses}

Time to event calculations were defined as time from start of HDR-SA brachytherapy to the relevant endpoints, and analyzed by Kaplan-Meier method. Event-free survival (EFS) was specified as time from start of HDR-SA brachytherapy to local-regional recurrence (LRR), distant metastasis (DM), or death. Overall survival was defined as time from start of HDR-SA brachytherapy to death or date last known alive. Patients without an event were censored at the date of last follow-up. The log-rank test was used to compare time from HDR-SA brachytherapy to an applicable event. Finally, univariate analyses were performed to determine potential predictors of outcomes (LRC, DM-free survival, and OS). Multivariate analyses were not performed due to small number of patients. A p-value of 0.05 was considered statistically significant for our analyses.

\section{Results}

\section{Patient, disease, and pre-brachytherapy treatment characteristics}

Characteristics of the 20 patients included in this study are described in Table 1. Angiosarcoma of the scalp or face was initially diagnosed for all patients between 2003-2017. The median age was 66 years (range, 45-85 years) and 15 of 20 patients $(75 \%)$ were male. The scalp was the primary site of disease in 15 of 20 cases $(75 \%)$, with multifocal disease being present in 10 of 20 patients (50\%).

Sixteen patients received HDR-SA brachytherapy as a part of their initial definitive cancer treatment, while four patients received brachytherapy at the time of disease recurrence or progression. Of patients who had brachytherapy as a part of their initial therapeutic strategy, 7 patients initially underwent surgical resection (treated between 2003 and 2012), 7 patients initially received chemotherapy (treated between 2008 and 2018, most commonly with a taxane-containing regimen), one patient received both surgery and chemotherapy before brachytherapy, and one patient had no previous treatment other than brachytherapy.

Four patients received brachytherapy for recurrent/ progressive disease, 1-7 years after other therapy. Two of these patients had progressed 1-2 years after chemotherapy alone and received brachytherapy at that time. In another two patients, brachytherapy was applied for in-field recurrences at 3 and 5 years after initial diagnosis. Both had undergone previous definitive surgery and radiation therapy, with radiation doses of 70.4 Gy (50.4 Gy followed by a $20 \mathrm{~Gy}$ boost with IMRT) and 61.2 Gy (with electrons), respectively. Prior to brachytherapy treatment, 3 patients had known lymph node involvement and 3 patients had known distant metastases (to lung or bone).

\section{Clinical outcomes}

Four patients did not complete the prescribed HDRSA brachytherapy course. Two patients interrupted the treatment early (at fraction 14 and 16, for a total dose of 
Table 1. Patient, disease, and pre-brachytherapy treatment characteristics

\begin{tabular}{|c|c|}
\hline & No. of patients \\
\hline \multicolumn{2}{|l|}{ Patient characteristics } \\
\hline \multicolumn{2}{|l|}{ Gender } \\
\hline Male & 15 \\
\hline Female & 5 \\
\hline \multicolumn{2}{|l|}{ Disease characteristics } \\
\hline \multicolumn{2}{|l|}{ Anatomic location } \\
\hline Scalp & 15 \\
\hline Face & 5 \\
\hline \multicolumn{2}{|l|}{ Focality } \\
\hline Unifocal & 10 \\
\hline Bifocal & 5 \\
\hline Diffuse & 5 \\
\hline \multicolumn{2}{|l|}{ Size } \\
\hline$<5 \mathrm{~cm}$ & 12 \\
\hline$\geq 5 \mathrm{~cm}$ & 8 \\
\hline \multicolumn{2}{|c|}{ Other disease pre-brachytherapy } \\
\hline$\underline{\text { Lymph node }+}$ & 3 \\
\hline Metastasis + & 3 \\
\hline \multicolumn{2}{|l|}{ Pre-brachytherapy treatment } \\
\hline \multicolumn{2}{|l|}{ Brachytherapy chronology } \\
\hline Initial definitive $t x$ & 16 \\
\hline Txat recurrence & 4 \\
\hline \multicolumn{2}{|l|}{ Chemotherapy } \\
\hline None & 10 \\
\hline Taxane only & 7 \\
\hline Taxane + gemcitabine & 3 \\
\hline \multicolumn{2}{|l|}{ Other local therapy } \\
\hline Surgery & 9 \\
\hline External beam RT & 2 \\
\hline
\end{tabular}

42 Gy and 48 Gy, respectively) due to skin toxicity. Two additional patients discontinued treatment in the context of failure to thrive (at fraction 5 and 7 , for a total dose on $15 \mathrm{~Gy}$ and $21 \mathrm{~Gy}$, respectively). Of note, the two patients who did not complete the prescribed treatment due to failure to thrive had the largest number of catheters, representing the largest involved disease fields (69 and 49, respectively).

The median follow-up from the start of brachytherapy was 45 months (range, 1-125 months). Clinical outcomes of this patients' cohort are presented in Table 2. Eight patients had a local disease recurrence ( 4 in-field and 4 at the margin). The 4 -year rate of local control was $63 \%$ (95\% CI: $45-91 \%$ ) (Figure 1A). Three patients recurred in the neighboring area $\geq 2 \mathrm{~cm}$ outside the field, and 3 patients recurred in lymph nodes only (parotid and/or neck). An additional 4 patients had both cutaneous and nodal recurrence ( 2 in-field, 1 marginal, and 1 out of field). Median time from the start of brachytherapy to LRR was
Table 2. Clinical outcomes and salvage

Local-regional recurrence

\begin{tabular}{ll}
\hline In-field & 4 \\
\hline Marginal & 4 \\
\hline Out-of-field & 3 \\
\hline Lymph node only & 3 \\
\hline Lymph node and cutaneous & 4 \\
\hline New metastasis (after brachy) & 5 \\
\hline Salvage treatment & 2 \\
\hline None (CMO) & 6 \\
\hline Chemo only & 2 \\
\hline Chemo $\rightarrow$ EBRT & 1 \\
\hline Chemo $\rightarrow$ brachy \#2 & 1 \\
\hline Chemo $\rightarrow$ EBRT $\rightarrow$ brachy \#2 & 1 \\
\hline Chemo $\rightarrow$ surgery $\rightarrow$ EBRT & 1 \\
\hline EBRT only & 2 \\
\hline Brachy \#2 only & 2 \\
\hline Status at last follow-up & 5 \\
\hline Alive no known recurrence & 2 \\
\hline Alive s/p RT recurrence & 11 \\
\hline Died no known recurrence & \\
\hline Died s/p RT recurrence & \\
\hline
\end{tabular}

brachy - brachytherapy, CMO - comfort measures only, chemo-chemotherapy, $\# 2$ - number 2, EBRT-external beam radiation therapy, $R T$-radiation

19 months (range, 1-60 months), with a 4-year local-regional control rate of 31\% (95\% CI: 11-53\%) (Figure 1B). Median progression-free survival (PFS) from the start of brachytherapy was 16.5 months, with a 4 -year PFS of $20 \%$ (95\% CI: 1-33\%) (Figure 1C).

Of the 16 patients with any disease recurrence or progression, 2 had no further therapy, 11 received chemotherapy ( 5 cases with additional radiation), and 3 patients received a second course of radiation therapy. Repeated brachytherapy alone was applied in 2 patients with marginal field recurrences, and after chemotherapy in 2 patients with out-of-field recurrences. The second course of brachytherapy was also prescribed to $51 \mathrm{~Gy}$ in 17 fractions. At the last follow-up, 2 patients were alive, and 2 patients had died with no evidence of recurrence. The median OS was 54 months, and 4-year OS was 54\% (95\% CI: 35-78\%) (Figure 1C).

For the 18 patients with acute skin toxicity, $78 \%$ $(n=14)$ had grade 3 dermatitis, and 22\% $(n=4)$ presented with grade 2 dermatitis. Moist desquamation was managed by cleaning the area with saline compresses, and using aquaphor mixed with $2 \%$ lidocaine jelly and/or xeroform gauze dressings. Ibuprofen 200-600 mg every 4-6 hours and/or tylenol 500-1,000 mg every 6-8 hours were also applied as needed. For the 10 patients for whom alopecia information was available, all were noted to have grade 1-2 alopecia. 

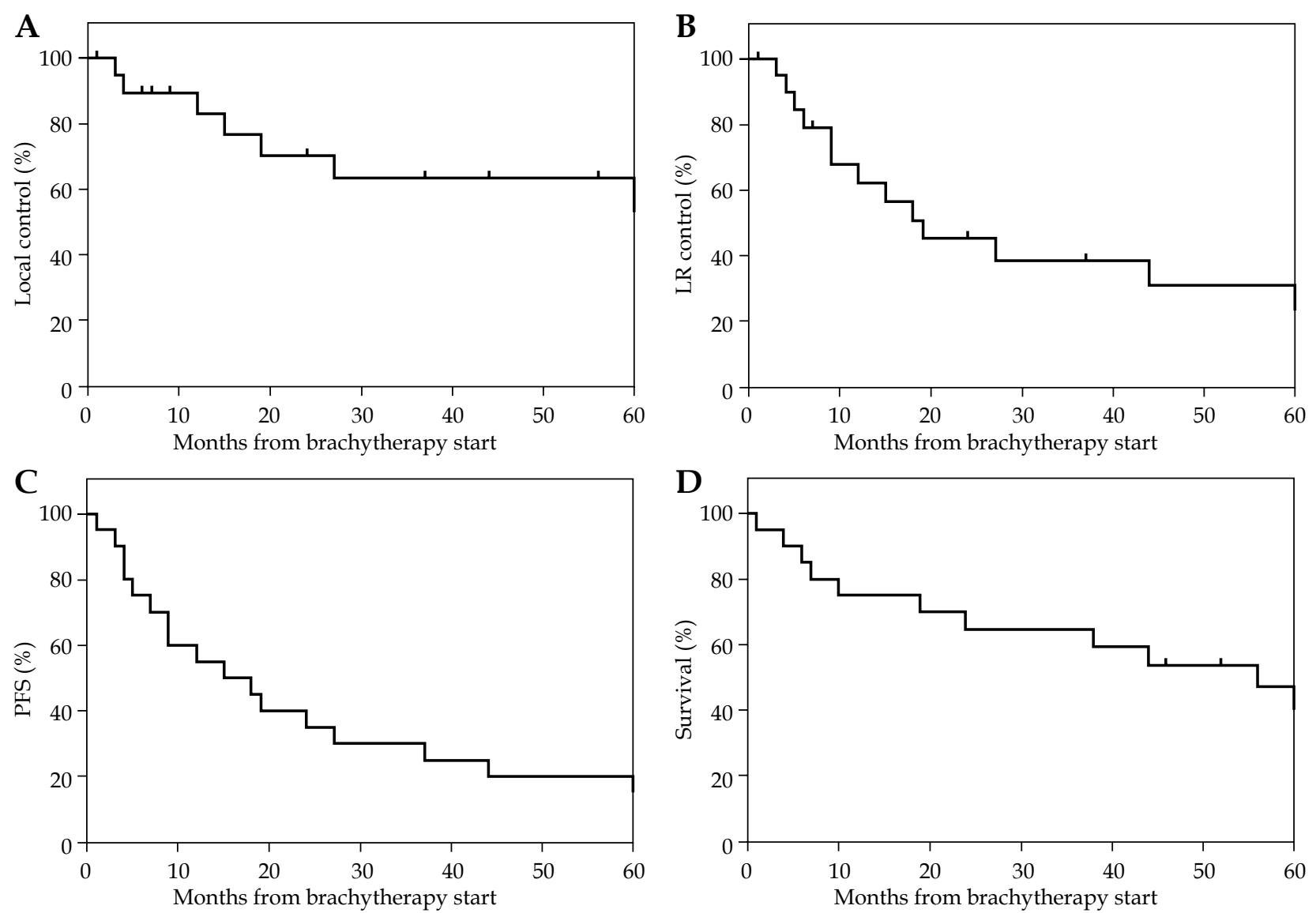

Fig. 1. Patient survival outcomes over time. A) Local control from the start of brachytherapy treatment. B) Local-regional control from the start of brachytherapy treatment. C) Progression-free survival of angiosarcoma patients from the start of brachytherapy treatment. D) Overall survival of angiosarcoma patients from the start of brachytherapy treatment

\section{Predictors of outcome}

On univariate analysis there was no difference in outcome by sex, focality (unifocal vs. multifocal), or relative timing of chemotherapy (Table 3). As others have shown, a primary anatomic location on the face was associated with better outcomes, including a significantly higher 4 -year LRC (75\% vs. $9 \%, p=0.042$; Figure $2 \mathrm{~A})$. No patients with primary tumors on the face developed DM at 4 years, while $55 \%$ of patients with scalp tumors did so. No association, however, was seen with anatomic site and OS. A smaller tumor size (primary disease $<5 \mathrm{~cm}$ ) was also associated with significantly better 4-year LRC $(35 \%$ vs. $0 \%, p=0.0099$; Figure $2 \mathrm{~B})$ and showed a trend towards better 4 -year OS (74\% vs. $25 \%, p=0.06)$, though had no association with DM. Patients who had surgery prior to HDR-SA brachytherapy (during the same treatment episode) had better outcomes across the board, including higher 4-year LRC ( $47 \%$ vs. $0 \%, p=0.0123$; Figure 2C), higher rates of being DM-free at 4 years $(64 \%$ vs. $37 \%, p=0.0407)$, and better 4 -year OS (70\% vs. $36 \%$, $p=0.0404)$. HDR-SA brachytherapy as a component of the initial treatment approach also resulted in better 4 -year LRC than its use as a component of salvage therapy at the time of disease progression or relapse (32\% vs. $0 \%, p=0.0084$; Figure 2D). This effect was also seen in
4 -year OS ( $66 \%$ vs. $0 \%, p=0.0019)$, but no difference was seen in DM rates. Due to small number of patients in this study, multivariate models were not constructed.

\section{Discussion}

Angiosarcoma of the scalp and face is a rare disease with poor prognosis and high rates of local and distant recurrence or progression. Our current institutional approach is multimodality therapy, typically starting with neoadjuvant chemotherapy (primarily taxane-based), followed by HDR-SA brachytherapy. For smaller lesions (typically $<2 \mathrm{~cm}$ ), surgery alone is sometimes considered. However, this practice has evolved over the past two decades and patients treated at our institution can be referred at a variety of timepoints during their treatment course. As a result, our institutional experience provides a unique opportunity to examine outcomes in the context of consistent use of HDR-SA brachytherapy, with some variability in the utilization of surgery and chemotherapy.

Prior to single-institution, retrospective studies have shown a benefit to radiation (typically as adjuvant therapy) with overall high recurrence rates. The UCLA experience found that 5-year disease-free survival was significantly higher in patients receiving surgery, followed by radiation vs. surgery alone ( $43 \%$ vs. 13\%). Five-year actu- 
Table 3. Univariate predictors of outcome

\begin{tabular}{|c|c|c|c|c|c|c|}
\hline Variable & $\begin{array}{c}\text { LRC } \\
\text { at } 4 \text { yrs }\end{array}$ & $\begin{array}{c}\mathrm{LRC} \\
p \text {-value } \\
\end{array}$ & $\begin{array}{l}\text { DM-free } \\
\text { at } 4 \text { yrs }\end{array}$ & $\begin{array}{l}\text { DM-free } \\
p \text {-value }\end{array}$ & $\begin{array}{c}\text { OS } \\
\text { at } 4 \text { yrs } \\
\end{array}$ & $\begin{array}{c}\text { OS } \\
p \text {-value }\end{array}$ \\
\hline \multicolumn{7}{|l|}{ Sex } \\
\hline Female & $36 \%$ & \multirow[t]{2}{*}{0.19} & $50 \%$ & \multirow[t]{2}{*}{0.507} & $58 \%$ & \multirow[t]{2}{*}{0.37} \\
\hline Male & $0 \%$ & & $30 \%$ & & $40 \%$ & \\
\hline \multicolumn{7}{|l|}{ Location } \\
\hline Face & $75 \%$ & \multirow[t]{2}{*}{$0.042^{*}$} & $100 \%$ & \multirow[t]{2}{*}{$0.0402^{*}$} & $60 \%$ & \multirow[t]{2}{*}{0.33} \\
\hline Scalp & $9 \%$ & & $45 \%$ & & $50 \%$ & \\
\hline \multicolumn{7}{|l|}{ Focality } \\
\hline Unifocal & $36 \%$ & \multirow[t]{2}{*}{0.28} & $47 \%$ & \multirow[t]{2}{*}{0.94} & $60 \%$ & \multirow[t]{2}{*}{0.82} \\
\hline Multifocal & $15 \%$ & & $64 \%$ & & $43 \%$ & \\
\hline \multicolumn{7}{|l|}{ Tumor size } \\
\hline$<5 \mathrm{~cm}$ & $35 \%$ & \multirow[t]{2}{*}{$0.0099^{\star}$} & $64 \%$ & \multirow[t]{2}{*}{0.49} & $74 \%$ & \multirow[t]{2}{*}{0.06} \\
\hline$\geq 5 \mathrm{~cm}$ & $0 \%$ & & $29 \%$ & & $25 \%$ & \\
\hline \multicolumn{7}{|c|}{ Previous chemotherapy } \\
\hline No & $36 \%$ & \multirow[t]{2}{*}{0.65} & $64 \%$ & \multirow[t]{2}{*}{0.21} & $57 \%$ & \multirow[t]{2}{*}{0.94} \\
\hline Yes & $21 \%$ & & $45 \%$ & & $52 \%$ & \\
\hline \multicolumn{7}{|c|}{ Surgery prior to brachy } \\
\hline No & $0 \%$ & \multirow[t]{2}{*}{$0.012^{\star}$} & $37 \%$ & \multirow[t]{2}{*}{$0.0407^{\star}$} & $36 \%$ & \multirow[t]{2}{*}{$0.040^{*}$} \\
\hline Yes & $47 \%$ & & $64 \%$ & & $70 \%$ & \\
\hline \multicolumn{7}{|c|}{ Brachy at progression } \\
\hline No & $32 \%$ & \multirow[t]{2}{*}{$0.0084^{*}$} & $58 \%$ & \multirow[t]{2}{*}{0.29} & $66 \%$ & \multirow[t]{2}{*}{$0.0019^{*}$} \\
\hline Yes & $0 \%$ & & $38 \%$ & & $0 \%$ & \\
\hline
\end{tabular}

$D M$ - distant metastasis, $L R C$ - loco-regional control, OS - overall survival

arial local control was $54 \%$ in patients who received adjuvant radiation vs. $19 \%$ in patients who had surgery alone [8]. The University of Michigan experience also showed an improved median OS in patients receiving surgery, followed by radiation vs. surgery alone (36.1 months vs. 9.2 months). Local recurrence patterns were not reported by treatment, but 17 of 29 patients developed local recurrence alone, while 4 additional patients developed local recurrence and distant metastasis [9]. The MD Anderson experience also found that combined modality therapy with surgery, followed by radiation was associated with improved OS, disease-free survival, and local control compared to treatment with either surgery or radiation alone. Specifically, the 3-year local control rate with surgery alone or radiation alone was 30\%, while the 3-year local control rate with surgery and radiation was $95 \%$ [6]. The British Columbia experience showed a trend to improved local relapse-free survival with surgery, followed by radiation vs. surgery alone (70\% vs. 40\%) [10]. Clinical outcomes in the current study were similar (with 4-year local control of 63\%, 4-year local-regional control of $30 \%$, and 4 -year PFS of $20 \%$ ), suggesting HDR-SA brachytherapy may not have significant efficacy advantages over other external beam approaches [3-7]. These disappointing results demonstrate a need for an intensified treatment approach. Given that the majority of patients developed in-field or marginal recurrences, one approach for patients who are not surgical candidates could be a larger initial external beam radiation therapy approach, followed by a brachytherapy boost to the area at highest risk.

In this cohort of patients, univariate analysis demonstrated several factors associated with outcome, which corroborate results of others. Specifically, disease location on the face (vs. scalp) was associated with better prognosis with a 4 -year LRC rate of $75 \%$ vs. $9 \%(p=0.042)$. A similar result was seen in an analysis from the University of Toronto, where 5-year LRC for the scalp vs. face was $53 \%$ vs. $9 \%(p=0.04)$ [19]. A meta-analysis of 11 studies including 379 patients also found that primary location of the scalp was associated with worse prognosis with a 5-year OS odds ratio (OR) of $3.089(p<0.001)$ [20]. This meta-analysis also found that larger tumor size $(\geq 5 \mathrm{~cm})$ was significantly associated with worse 5-year OS (OR = 3.566, $p=0.013$ ), which is similar to the trend found in the current study, with a 4 -year OS difference of $74 \%$ vs. $25 \%(p=0.06)$ and a significant difference in 4-year LRC of $35 \%$ vs. $0 \%(p=0.0099)$ based on size. While surgery prior to HDR-SA brachytherapy was associated with better outcomes in our study, this was likely due to the fact that typically, more favorable lesions were treated with surgery. In particular, definitive HDR-SA brachytherapy alone is more often used for lesions spanning a larger area, which we and others have found associated with worse prognosis. In addition, this study found that salvage HDR-SA brachytherapy was associated with worse prognosis than HDR-SA brachytherapy as a component of therapy at initial diagnosis. This difference likely rep- 


\section{A}

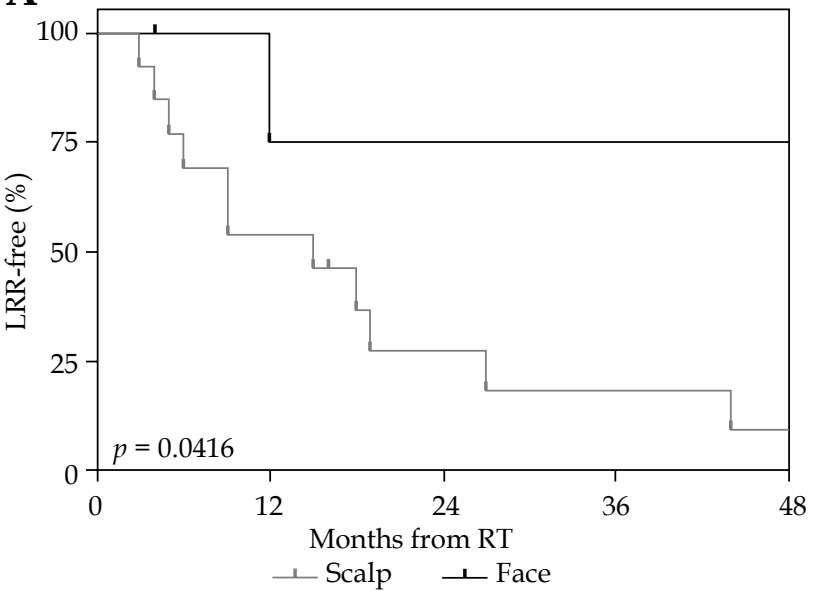

C

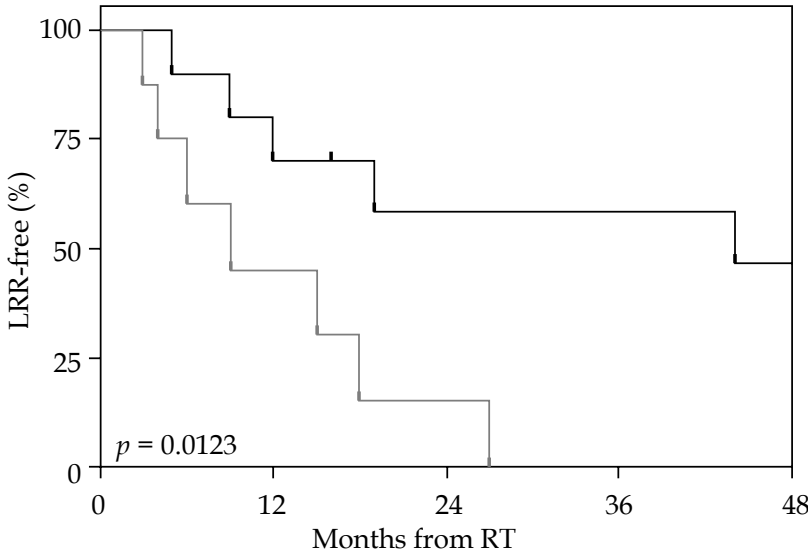

B

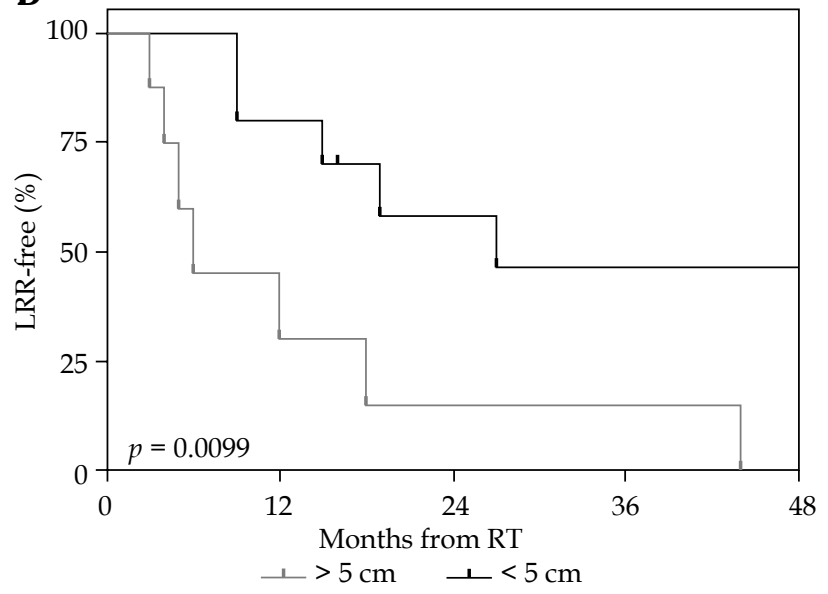

D

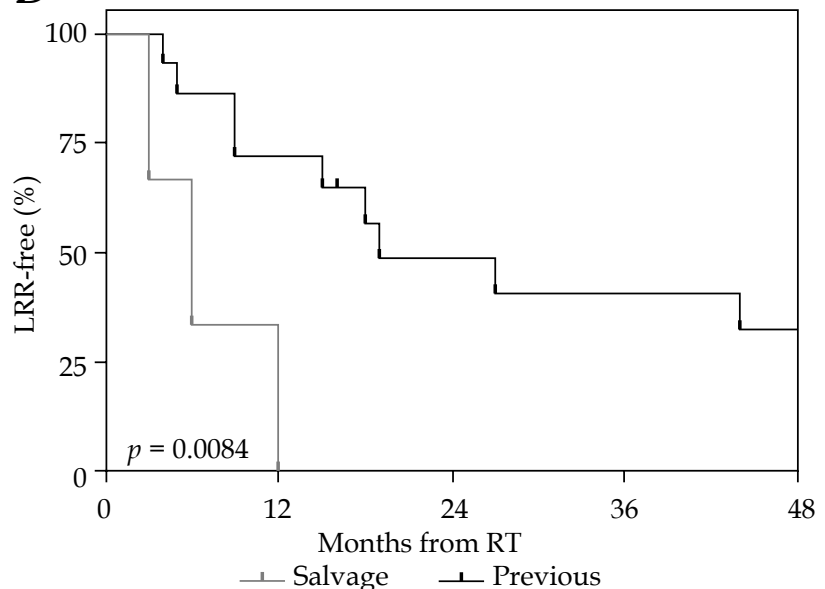

Fig. 2. The effect of disease and treatment variables on loco-regional recurrence. A) Loco-regional recurrence by anatomic location (scalp vs. face). B) Loco-regional recurrence by tumor size ( $>5 \mathrm{~cm}$ vs. $<5 \mathrm{~cm}$ ). C) Loco-regional recurrence by use of prior surgery (definitive vs. salvage). D) Loco-regional recurrence by prior disease progression (salvage vs. previous)

resents the particularly challenging nature of managing progressive or recurrent angiosarcoma, regardless of therapeutic modalities used.

Limitations of this study include its retrospective nature, prone to selection bias. There was also treatment heterogeneity with respect to the use of surgery and systemic therapy. Lastly, the sample size was small. Despite these limitations, given the rarity of cutaneous angiosarcoma of the face and scalp, the findings are informative.

In conclusion, cutaneous angiosarcoma of the face and scalp treated with HDR-SA brachytherapy was associated with moderate local control rates, which are similar to other radiation treatment techniques used either alone or in combination with surgery. Tumors involving the face performed better than those of the scalp. Progression-free survival was poor, and treatment intensification strategies are needed.

\section{Disclosure}

The authors report no conflict of interest.

\section{References}

1. Young RJ, Brown NJ, Reed MW et al. Angiosarcoma. Lancet Oncol 2010; 11: 983-991.

2. Albores-Saavedra J, Schwartz AM, Henson DE et al. Cutaneous angiosarcoma. Analysis of 434 cases from the Surveillance, Epidemiology, and End Results Program, 1973-2007. Ann Diagn Pathol 2011; 15: 93-97.

3. Cassidy RJ, Switchenko JM, Yushak ML et al. The importance of surgery in scalp angiosarcomas. Surg Oncol 2018; 27: A3-A8.

4. Köhler HF, Neves RI, Brechtbühl ER et al. Cutaneous angiosarcoma of the head and neck: report of 23 cases from a single institution. Otolaryngol Head Neck Surg 2008; 139: 519-524.

5. Buschmann A, Lehnhardt M, Toman N et al. Surgical treatment of angiosarcoma of the scalp: less is more. Ann Plast Surg 2008; 61: 399-403.

6. Guadagnolo BA, Zagars GK, Araujo D et al. Outcomes after definitive treatment for cutaneous angiosarcoma of the face and scalp. Head Neck 2011; 33: 661-667.

7. Patel SH, Hayden RE, Hinni ML et al. Angiosarcoma of the scalp and face: the Mayo Clinic experience. JAMA Otolaryngol Head Neck Surg 2015; 141: 335-340.

8. Mark RJ, Poen JC, Tran LM et al. Angiosarcoma. A report of 67 patients and a review of the literature. Cancer 1996; 77: 2400-2406. 
9. Pawlik TM, Paulino AF, McGinn CJ et al. Cutaneous angiosarcoma of the scalp: a multidisciplinary approach. Cancer 2003; 98: 1716-1726.

10. Jasper KD, Holloway CL, DeVries KJ et al. Local relapse and survival outcomes in patients with scalp sarcoma: a retrospective study of 95 patients treated in a provincial cancer care institution over 25 years. Cureus 2019; 11: e5236.

11. Akazawa C. Treatment of the scalp using photon and electron beams. Med Dosim 1989; 14: 129-131.

12. Tung SS, Shiu AS, Starkschall G et al. Dosimetric evaluation of total scalp irradiation using a lateral electron-photon technique. Int J Radiat Oncol Biol Phys 1993; 27: 153-160.

13. Wojcicka JB, Lasher DE, McAfee SS et al. Dosimetric comparison of three different treatment techniques in extensive scalp lesion irradiation. Radiother Oncol 2009; 91: 255-260.

14. Ostheimer C, Hübsch P, Janich M et al. Dosimetric comparison of intensity-modulated radiotherapy (IMRT) and volumetric modulated arc therapy (VMAT) in total scalp irradiation: a single institutional experience. Radiat Oncol J 2016; 34: 313-321.

15. Kai $Y$, Toya R, Saito $T$ et al. Plan quality and delivery time comparisons between volumetric modulated arc therapy and intensity modulated radiation therapy for scalp angiosarcoma: A planning study. J Med Radiat Sci 2018; 65: 39-47.

16. Sanada T, Nakayama H, Irisawa R et al. Clinical outcome and dose volume evaluation in patients who undergo brachytherapy for angiosarcoma of the scalp and face. Mol Clin Oncol 2017; 6: 334-340.

17. Szlag M, Wojcieszek P, Kellas-Ślęczka $S$ et al. Individual multi-catheter mould technique in high-dose-rate brachytherapy - personalized approach in treating multifocal angiosarcoma of the face. J Contemp Brachytherapy 2019; 11: 337-342.

18. Mitra D, Pei Y, Buzurovic I et al. Angiosarcoma of the scalp and face: a dosimetric comparison of HDR surface applicator brachytherapy and VMAT. Sarcoma 2020; 2020: 7615248.

19. Bernstein JM, Irish JC, Brown DH et al. Survival outcomes for cutaneous angiosarcoma of the scalp versus face. Head Neck 2017; 39: 1205-1211.

20. Shin JY, Roh SG, Lee NH et al. Predisposing factors for poor prognosis of angiosarcoma of the scalp and face: Systematic review and meta-analysis. Head Neck 2017; 39: 380-386. 